\title{
Characterization of Si Mid-infrared Photonic Components for Chemical and Gas Sensing
}

\author{
Ursula Hedenig ${ }^{* 1}$, Thomas Grille ${ }^{1}$, Grant A.D. Ritchie ${ }^{2}$, James M. Kirkbride ${ }^{2}$, Bernhard Jakoby $^{3}$ and \\ Ventsislav Lavchiev ${ }^{3}$ \\ ${ }^{1}$ Infineon Technologies Austria AG, Siemensstrasse 2, 9500 Villach, Austria, \\ ursula.hedenig@infineon.com \\ ${ }^{2}$ Department of Chemistry, The Physical and Theoretical Chemistry Laboratory, University of Oxford, \\ South Parks Road, Oxford, UK, OX1, 3QZ \\ ${ }^{3}$ Institute for Microelectronics and Microsensors, Johannes Kepler University, Altenbergerstrasse 69, \\ 4040 Linz, Austria
}

\begin{abstract}
:
The paper discusses how, using proper design and modelling, IR-absorption sensors could achieve appropriate detection sensitivity, with micron- and submicron dimensions and to be integrated within a single-platform device. Detailed material and structure characterization along with the employed technology is demonstrated for the recently fabricated prototypes. In our device concept, a MIR source emits electromagnetic (EM) field, coupled with a Si waveguide (WG). The latter operates as an interaction volume leading to partial absorption of energy in the sample yielding an attenuation of the guided wave. It is detected at the output with a MIR detector. By careful choice of the waveguide thickness, it is possible to change the detector's sensitivity. There is an interaction of the thickness and the absorption by coated fluid. In case of a rib WG or the characteristic geometry dimensions in a photonic crystal (PhC) WG the desired spectral range of operation can be obtained by proper tuning the waveguide width and height. To evaluate the device performance, photonic simulations with the finite difference time domain (FDTD) method and beam propagation method (BPM) in 3D are applied. These are powerful methods, which verified the guiding EM modes, the attenuation of the field along the WG and resulted in optimization of the device dimensions. The developed Si photonic components, are suitable for integration into a miniaturized sensor system and operates in the wavelength region $\lambda=3-7 \mu \mathrm{m}$. This sensor configuration is compatible to the Si technology and can also be realized on a single chip. In addition, the principle of operation is not limited to a single wavelength. It can be applied to a broad spectral range too.
\end{abstract}

Key words: mid-infrared, spectroscopy, ellipsometry, m-line, photonic crystals, chemical sensing, Si, photonics.

\section{Introduction}

The sensor technology can be classified as one of the fields, which has experienced the most extensive developing in the past decade. Generally there are physical, chemical, gas and biological sensors. The basic directions in the research and developing of sensors are introduction of new concepts and new materials. These ideas have led to emerging of many novel sensors: magnetic sensors and magnetometers measuring strength and direction of magnetic fields; absorption sensors measuring changes in absorption spectrum of gases and liquids; surface plasmon resonance sensors for chemical sensing, physical quantities detection, biosensing (biomolecular interactions), humidity; imaging sensors as CCD and magnetic resonance imaging MRI; gas sensors for observation and control of gas emissions and gas presence in the environment; LIDAR (light detection and ranging) for imaging objects; tomography sensors (optical coherence tomography and positron emission tomography); electronic noses measuring odors and flavors etc. Different materials are in use for varying sensor types.

Chemical monitoring is one of the special interests addressing e.g. pollutants detection (especially in water), with the aim of improving the protection of human health and the environment through the better and earlier identification of the properties of chemical substances. In this field, the demand for devices allows a faster, easier, real-time analysis and on-line, in situ detection. At the moment a long complex protocol analysis performed by specialized laboratories. It is increasing because the concern for the 
environment and sustainability issues are spreading. Distinctive interest is associated with the photonic sensors and the related quantum dots (QDs) as emitters and detectors.

Fluid composition can be efficiently analyzed in terms of characteristic absorption in the IR range. The MIR range is particularly interesting as most molecules feature characteristic absorption "fingerprints" there. Integrated sensors tackling measurement is rarely available.

We present Si-based absorption sensor for fluids which is suitable for integration into a single-platform sensor system. The sensor is designed to operate in the MIR region ranging in the wavelengths $\lambda=3 \mu \mathrm{m}$ to $\lambda=7 \mu \mathrm{m}$. We present the design, the modelling and the optical characterization of the used materials. The sensor is designed as a single-mode Si waveguide (WG) on low refractive index $\mathrm{Si}_{3} \mathrm{~N}_{4} / \mathrm{SiOx} / \mathrm{Si}_{3} \mathrm{~N}_{4}$ membrane. The single-mode requirement for the WG is needed to avoid losses due to imperfections on the WG walls causing redistribution of the carried energy among the different modes. The waveguide interacts with the fluid sample which results in an evanescent field extending into the sample. In addition, the principle of operation is not limited to a single wavelength: by changing the waveguide dimensions, it can be applied to a broad spectral range. In this case with its dimensions, performance and Si-compatibility, the sensor is expected to pass previously published devices with planar waveguide geometry.

\section{Design and Modelling: Spectroscopic Ellipsometry and 3D Photonic Simulations}

The photonic structures have been fabricated from polycrystalline $\mathrm{Si}$ on $\mathrm{Si}_{3} \mathrm{~N}_{4} / \mathrm{SiOx} / \mathrm{Si}_{3} \mathrm{~N}_{4}$ a substrate. All the layers have been grown by low pressure chemical vapour deposition (LPCVD). Knowledge of the complex dielectric functions of the materials is of fundamental importance for photonic applications. For this purpose, variable angle spectroscopic ellipsometry [1] has been developed as a tool to obtain the dielectric function of poly-Si, $\mathrm{Si}_{3} \mathrm{~N}_{4}$ and SiOx. For the proper characterization, separate layers of $\mathrm{Si}, \mathrm{Si}_{3} \mathrm{~N}_{4}$ and $\mathrm{SiOx}$ have been deposited on Si substrates.

The ellipsometry measurements have been carried out in the infrared spectral range $\lambda \approx 1.26-38 \mu \mathrm{m}$. Part of the spectra are demonstrated in Fig. 1, which shows the dielectric functions of the materials obtained through modeling of the experimental data.

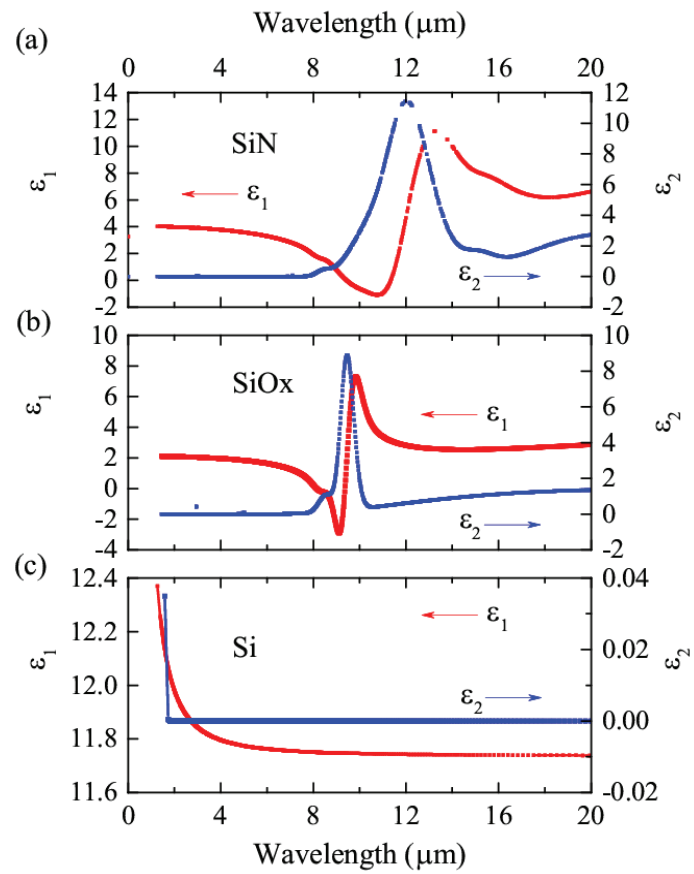

Fig. 1. The dielectric functions of the used materials obtained via spectroscopic ellipsometry.

Figure 1 demonstrates that the materials are optically transparent up to $\lambda \approx 8 \mu \mathrm{m}$ and may be implemented for photonic simulations.

Photonic simulations were done next. Rib waveguides (WGs) and photonic crystal waveguides (WGs) were investigated. The dielectric functions of the materials acquired from the ellipsometry served as input parameters for the photonic simulations. By implementing the beam propagation method (BPM) in 3D, it was possible to assure the guiding performance of the structures, to evaluate the guided EM modes and to estimate the structure's dimensions in order to obtain low-mode performance. On the other hand, the finite difference time domain (FDTD) method combined with the BPM allowed an evaluation of the sensitivity of the device. Finally, simulations for calculation of the band structure of the photonic crystals (PhC) and the characteristic dimensions of the PhC to obtain a photonic band gap in the spectral range of interest were done.

The single-mode requirements lead to WG dimensions of $2 \mu \mathrm{m}$ width $\times 600 \mathrm{~nm}$ height for an operation at $\lambda=5.5 \mu \mathrm{m}$. Simulation results with 3D BPM are shown on Fig. 2. The WG will support two TE modes $\left(\mathrm{TE}_{0}\right.$ and $\left.\mathrm{TE}_{1}\right)$ and one TM mode $\left(\mathrm{TM}_{0}\right)$ at a wavelength of $\lambda \approx 5.3 \mu \mathrm{m}$ (at $\lambda \approx 6 \mu \mathrm{m}$ the guided modes are already $\mathrm{TE}_{0}$ and $\mathrm{TM}_{0}$ only). 


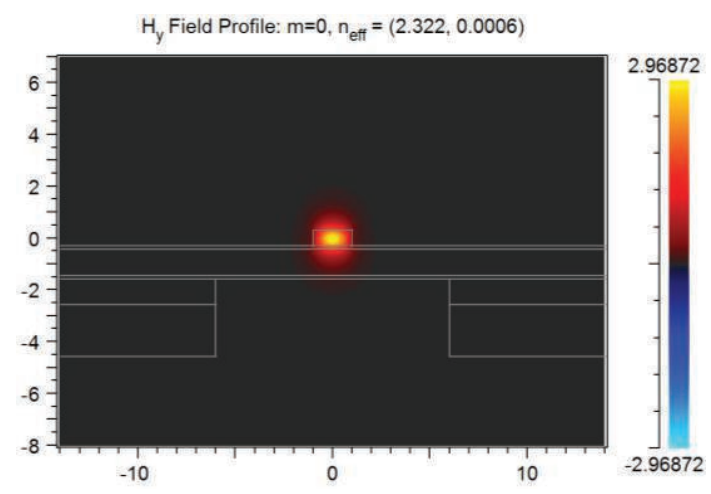

$H_{y}$ Field Profile: $m=1, n_{\text {eff }}=(1.367,0.0038)$

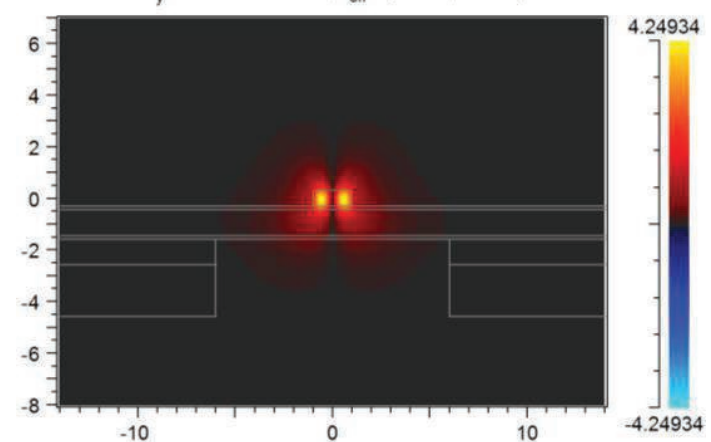

$H_{x}$ Mode Profile $m=0, n_{\text {eff }}=(1.37,0.0018)$

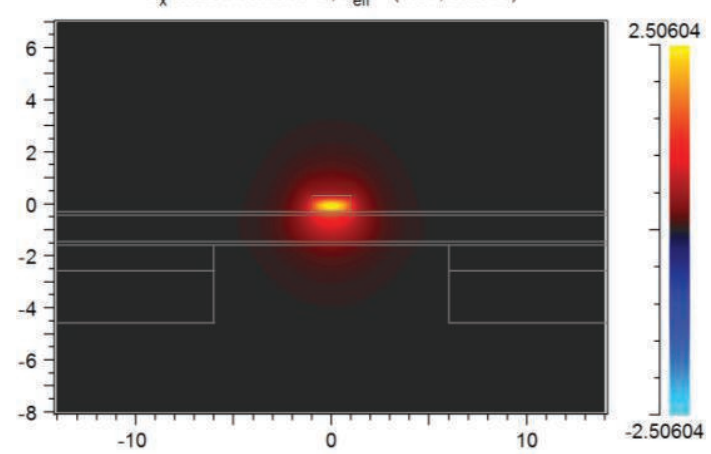

Fig. 2. The H-field of the guided waves in a rib WG with a cross section of $0.6 \times 2 \mu \mathrm{m}$. The $T E_{0}$ and $T E_{1}$ modes are the topmost and the middle; the TMO mode is on the bottom.

Figure 3 shows the projected band structure of a photonic crystal structure over the full Brilluin zone. The $\mathrm{PhC}$ is like holes in a Si slab filled with SiOx (TEOS). Characteristic dimensions of the investigated $\mathrm{PhC}$ are a periodicity of the holes $a=1.5 \mu \mathrm{m}$ and radius $\mathrm{R}=0.6 \mu \mathrm{m}$. A couple of partial photonic band gaps can be noted under the light line including one at a wavelength $\lambda \approx 5.5 \mu \mathrm{m}$.

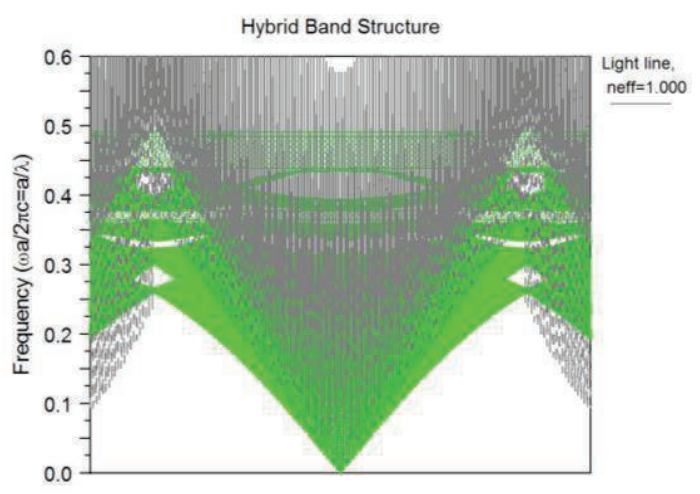

Fig. 3. Projected band structure of PhC with a period $a=1.5 \mu \mathrm{m}$ and radius $R=0.6 \mu \mathrm{m}$.

Based on these designs, $\mathrm{Si}$ waveguides have been fabricated, which will be demonstrated further in the paper.

\section{Fabrication}

The manufacturing starts with deposition of a bi-layer of $\mathrm{SiOx} / \mathrm{SiN}$ on 8" Si wafers (step 1). The nitride layer is deposited by LPCVD technique to obtain the stoichiometry $\mathrm{Si}_{3} \mathrm{~N}_{4}$. The bottom SiOx layer is used as an etch-stop for the Bosch etching process from the backside. On top, a polycrystalline $\mathrm{Si}$ layer is deposited, which serves for waveguiding. The PhC structures were done by deep-UV lithography (exposure wavelength $\lambda=193 \mathrm{~nm}$, Canon FPA6300ES6a scanner, Canon FPA-555iZ stepper) in the top Si layer and a following plasma etch process (step 2). Next process is the deposition of SiOx filling the PhC holes (step 3). A Chemical-mechanical polishing was carried out to remove the SiOx above the Si layer and the oxide layer on the backside (step 4). Finally, chemical etching onto the backside of the sample forms a membrane, on which the photonic crystal remains (step 5). Samples with a solid substrate by omitting step 5 were fabricated as well. The rib-waveguide structure is manufactured in a similar process. The substrate is a $\mathrm{SiN} / \mathrm{SiO}_{2} / \mathrm{SiN}$ membrane of thickness $80 \mathrm{~nm} / 1000 \mathrm{~nm} / 80 \mathrm{~nm}$. The nitride is selected due to its optical transparency in the mid-infrared [2] and its low refractive index. A thick membrane purely made from SiN was difficult to achieve due to the accumulation of stress in the layer and the associated cracking. SiOx is implemented in the substrate and the filling because of its low refractive index in MIR and the possibility to polish. 

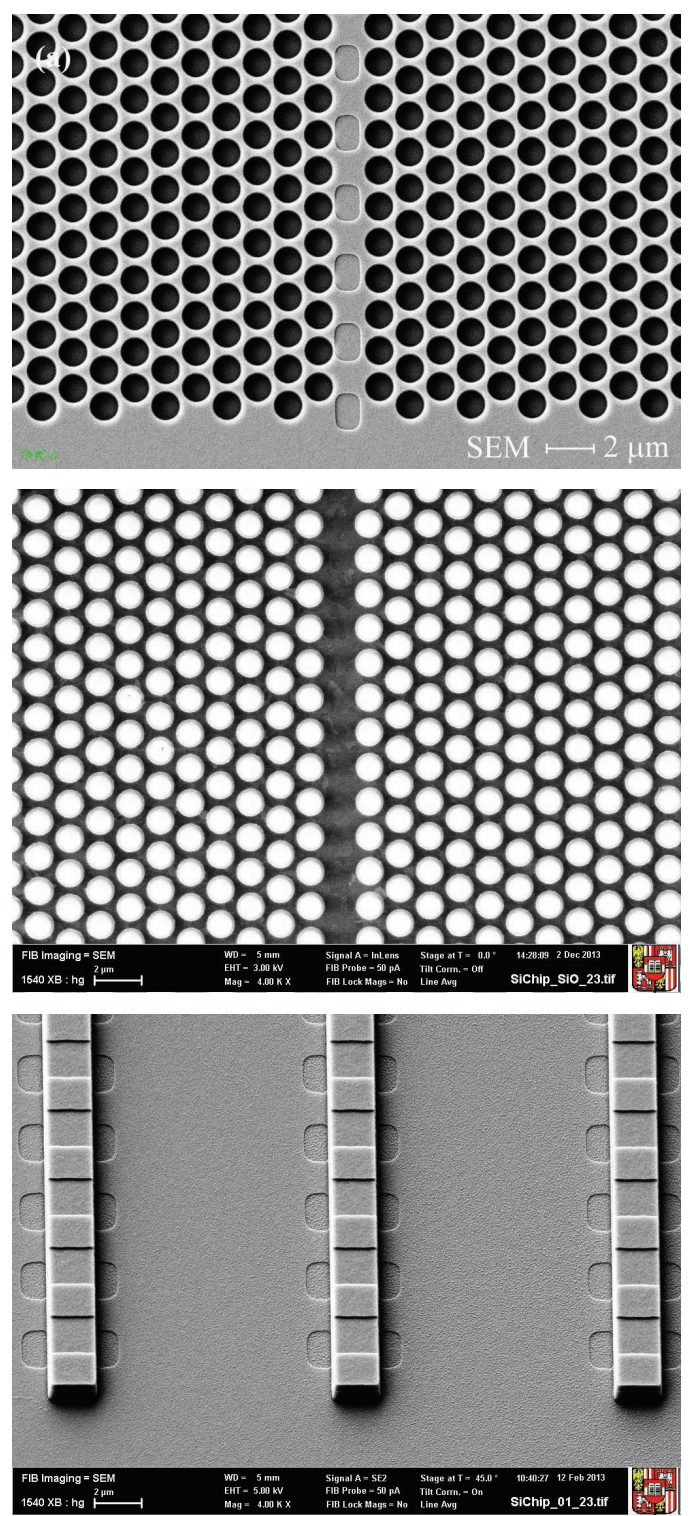

Fig. 4. SEM images of photonic crystal structures a) with air holes in Si (topmost; the PhC WG contains grating for input and output coupling of light; b)with a SiOx filling of the holes (middle); the bright contrast is achieved due to the electron charge accumulated in the oxide layer; c) rib WG structure.

\section{M-line Characterization of the Waveguides}

The fabricated photonic crystals and rib waveguides had been characterized in a series of transmission spectroscopy and m-line experiments.

The guiding behavior of the waveguides can be investigated through the m-line spectroscopy. This is the most efficient technique, which allows for direct observation of the guided electromagnetic modes in a waveguide first introduced by R. Ulrich and P. K. Tien in the early 70 s $[3,4]$.
The m-line spectroscopy was done by developing a tunable quantum cascade laser source QCL (Daylight Solutions, 21052-MHF). The laser operates with a continuous tuning wavelength range from $1776 \mathrm{~cm}-1$ to $1968 \mathrm{~cm}-$ $1(\lambda=5.08-5.63 \mu \mathrm{m})$ and a power output $P \max =140 \mathrm{~mW}$. The output beam from the PhC waveguide is detected by thermoelectrically cooled HgCdTe (MCT) detector (PVI-2TE-6, Vigo System S.A.). Measurements with both TE and TM polarization of the laser beam were done. The beam coupling in and out of the WGs was accomplished by Ge prisms.

Figure 5 shows the results on m-line spectroscopy with both rib waveguides and photonic crystal waveguides. In such experiments, the laser beam is coupled into the Si WG by a Ge prism, which has a refractive index higher than Si. The angle of incidence is varied so that the k-vector of the incident beam is matching a k-vector of a possible mode in the waveguide. In certain angle of incidences $\theta_{3}$, with fulfilling conditions a mode is excited. Further, the mode is coupled out of the waveguide by the same prism. The signal at the detector at the output is collected as a function of the angle of incidence.

As a comparison, structures on a membrane and on a solid substrate were investigated. The behavior of the structures is similar in both cases, but the waveguides show modes excited at different angles of incidence. This corresponds to modes with different k-vector e.g. with different effective refractive index $n_{\text {eff }}$. This effect could be explained with the influence of the air substrate below the membrane, which is only $1200 \mathrm{~nm}$ thick (comparing to the $600 \mathrm{~nm}$ thickness of the waveguide). Slightly different pressure on the $\mathrm{Ge}$ prism toward the $\mathrm{Si}$ waveguide may also lead to different coupling behavior from the prism to the WG $[3,4]$.

FDTD simulations on m-line spectroscopy confirm the m-line experiments. Fig. 6 demonstrates the simulation results with TE polarized light. In the simulations, the source of EM radiation is placed before the Ge prism and the detector is placed in the waveguide (right end on the figure) at sufficient distance so that the scattered light from the prism does not enter the detector. The angle of incidence Phi in the simulation corresponds to the angle $\theta_{3}=51^{\circ}$ in the prism and in the experiments. 
(a)

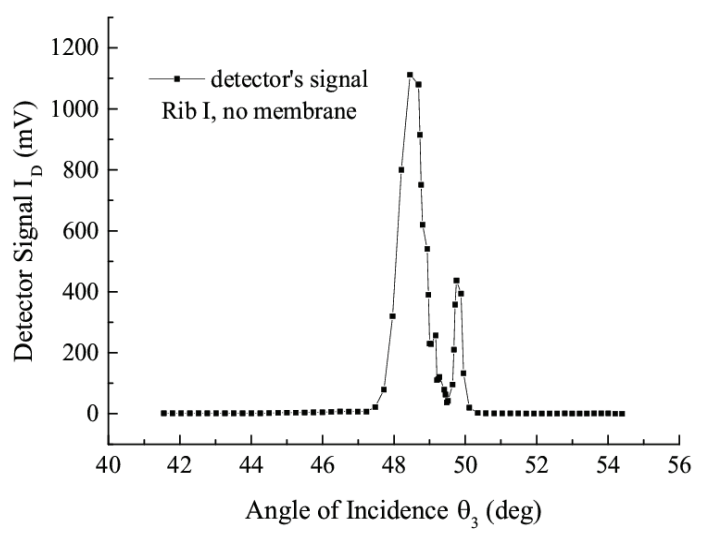

(b)

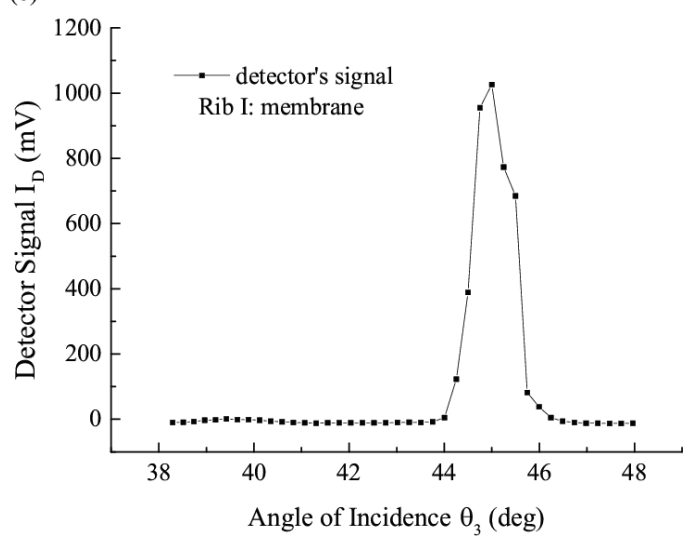

ə)

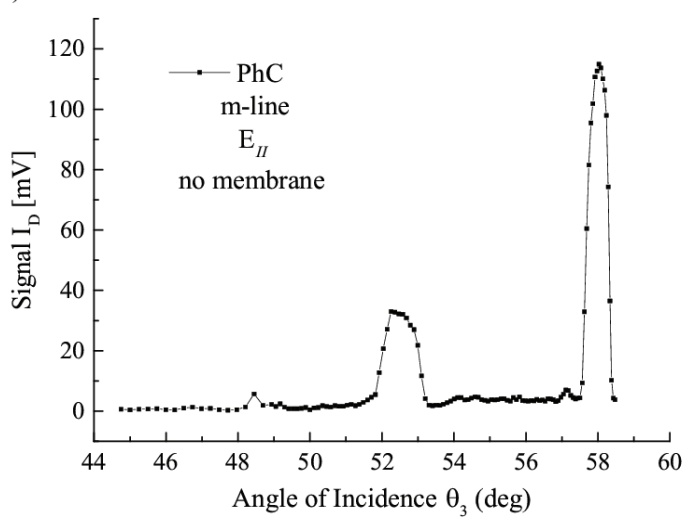

Fig. 5. M-line spectroscopy on PhC and rib waveguides with TE polarized laser beam. The peaks illustrate the excited modes.

\section{Conclusions}

In this paper, we presented Si rib waveguides and photonic crystals. The structures are designed for operation in the spectral range $\lambda=3 \mu \mathrm{m}$ to $\lambda=7 \mu \mathrm{m}$. The design and the modelling of these passive components were done by the finite difference time domain (FDTD) method and the beam propagation method (BPM). The two tools constitute powerful methods for modeling of the propagating EM modes, the attenuation of the

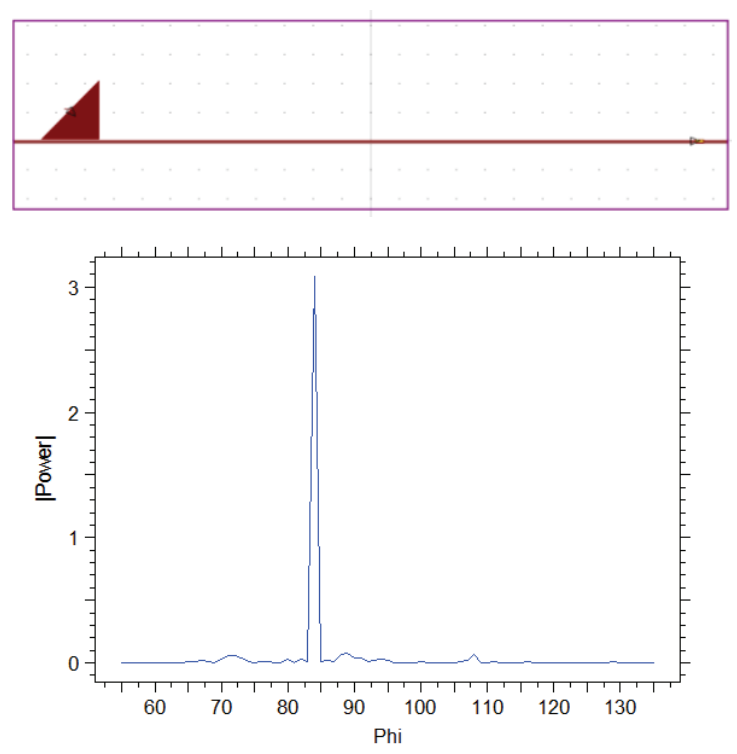

Fig. 6. FDTD simulation of $m$-line experiment. The excitation wavelength is set to $5.3 \mu \mathrm{m}$.

field along the WG and optimization of the device dimensions. The guiding behavior of the waveguides (photonic crystal and rib) has been investigated and proved via $m$-line spectroscopy at wavelength $5.3 \mu \mathrm{m}$ with a QCL laser. Two modes can be excited in such waveguides. The demonstrated structures can be successfully implemented for absorption sensors in the MIR range for chemicals and gases.

\section{Acknowledgement}

This work has been supported by the Austrian COMET-K2 program of LCM, and was funded by the Austrian federal government and the federal state of Upper Austria. The authors express gratitude to Prof. Wolfgang Jantsch for various useful discussions and to Günter Hesser at ZONA for his SEM microscopy support. The authors express special thanks to Prof. Gus Hancock from University of Oxford for his support in various experimental and organizational issues. Additionally the knowledge of the MEMS Team of Infineon Technologies Austria AG enabled the successful results of this work.

\section{References}

[1] H. Fujiwara, Spectroscopic ellipsometry Principles and applications (John Wiley \& Sons, Ltd., West Sussex 2007).

[2] R. Soref, Nat. Photonics, 2010, 4, 495-497.

[3] R. Ulrich, J. Opt. Soc. Am., 1970, 60, 1337-1350.

[4] P. K. Tien and R. Ulrich, J. Opt. Soc. Am., 1970, $60,1325-1337$. 